\title{
Approaching a Fair Deal for Significance and Other Concerns
}

Inaugural Editorial, Journal of Experimental Social Psychology

Roger Giner-Sorolla, University of Kent (version of 26 January 2016)

As I assume the Editor-in-Chief position at the Journal of Experimental Social Psychology, my first thanks are due to the preceding Chief Editor, Laurie Rudman, who has been wonderfully supportive and insightful during my term as Associate Editor and in the transition period. Likewise, I want to extend my gratitude in introducing the new editorial team: Senior Associate Editor Antonio Freitas and Associate Editors Sarah Gervais, Ursula Hess, Tara MacDonald, Sean McCrea, Nicole Mead, Elizabeth Page-Gould, Nicholas Rule, Shaul Shalvi, Robbie Sutton, and Michael Wohl. The support team at Elsevier, including Adam Fraser, Caroline Jones, and Karen Villahermosa, has also been very helpful in managing the challenges of a new and improved online editorial system. Last but not least, I very much appreciate the support of our sponsor organization, the Society for Experimental Social Psychology, and of its Publication Committee.

Although new editorial guidelines for the journal have already been posted, I would like to reiterate that JESP is still primarily looking for manuscripts that report original research that takes an experimental approach relevant to social psychology. This does not mean that every study in the article needs to be experimental; but at some point the article should draw upon the method that gives the journal its name. Also, quasi-experimental and intervention research articles are also welcome, if grounded in theory and otherwise 
appropriate for the journal. We are also increasingly interested in articles that improve methodological or statistical understanding about the research we do, especially if written accessibly and accompanied by usable techniques and tools. Some examples of topics that might be welcome, by no means an exhaustive list, are: developing Gabriel's (1978) confidence intervals, with their readily interpreted overlap, to cover cases of unequal variance; explaining and interpreting effect sizes in social psychology; principled methods of dealing with non-normal data; clarifying the role of experimental technique and setting in lab studies.

What do experimental social psychologists want when they submit their manuscript to a journal? They want the journal to be good, of course; to publish good work and thereby to validate their own work if it is chosen for publication. But they also want the process to be fair. Our papers should be judged by well-explained standards, using flexible but broadly consistent procedures. The process should respect the time course of research careers and the timeliness of research.

In taking on the Chief Editorship of JESP I have had a discussion with incoming Associate Editors, and some outgoing ones, about what they think is reasonable in the editorial process. This discussion, of course, takes place against a backdrop of ferment in the field of psychology. For the past four years or so, changes in the standards of statistical evidence have been much talked about and, increasingly, implemented. While most prospective JESP authors are probably quite familiar with the background of these issues, those who are curious may refer, for example, to the two special sections of Perspectives on Psychological 
Science in November 2012 and May 2014, or to a valedictory editorial in that journal which succinctly and evenhandedly traces the trajectory of the methods "revolution" (Spellman, 2015).

Most importantly for a journal editor, these changes have brought about great uncertainty about how any given way of reporting research will be evaluated. Authors become more cautious. Those who misjudged editorial standards feel unfairly treated. To resolve this uncertainty, a journal needs to balance visible and foreseeable standards with the flexibility to implement them fairly. This means explaining the logic and intent behind standards, and making sure that the Associate Editors who handle manuscripts know them and agree in broad terms. In the rest of this Editorial I will explain the underlying reasons in support of a number of new policies at JESP.

\section{Timeliness of the review process}

Publishing in our field can take a long time. The passing months are especially precious to early-stage researchers facing the job market or the tenure clock. Some reasons for this are beyond the power of a single journal editor to address. However, at JESP and other journals, I have found useful the suggestion of Hewstone and Stroebe (2008) to limit reviewers' involvement in the evaluation of revised manuscripts. Endless rounds of revisions, shifting goalposts as new reviewers and objections enter the fray: all these practices delay the dissemination of our science and reduce the perception of a fair deal. 
Another unfortunate phenomenon is the often long delay between the collection of data in the immediate wake of an historical event and its eventual publication -- sometimes three, four or five years down the line. FlashReports at JESP have been established as an expedited form of short article, with the aim of offering fast review on "topics of timely or historical importance." Most incoming and outgoing editors agreed that the brief format should not interfere with the completeness of reporting and rigor of evidence usually required of a JESP contribution (cf. Ledgerwood \& Sherman, 2012). Faster publication turnaround is also something that could benefit research in general, whether or not it bears a visible timestamp from a memorable event.

\section{Standards of evidence}

Authors also expect to know in broad terms how their article's claim for evidence is evaluated. To explain our emerging policy on statistical and methods reporting, it is useful to paint a picture of the publishing standards that were commonly held by many in social psychology prior to 2011, but which have come under increasing criticism since then. I will characterize this set of standards by its own logic, in broad strokes, and in a spirit of understanding rather than blame. Much of the recent discussion of statistics and methods has addressed the limitations of these rules of publishing, even though there is still much in them worth keeping.

For most well-regarded empirical outlets, the previous standards required an article to:

1. Propose an innovative idea that can be confirmed by an empirical effect; 
2. Report one or more studies, each with a statistically significant positive result supporting the focal effect(s);

3. Defend the interpretation of these positive results against alternative explanations: for example, rival theories or method artifacts.

Limitations of relying on novelty. To mention a much-remarked limitation of the first of these standards, direct replication studies based on other researchers' work were previously avoided for failing the "innovative" requirement. More recently, they have become better supported. Along with a number of other journals, JESP has already announced its openness to such efforts and endorsed a preliminary set of standards for evaluating them (IJzerman, Brandt et al., 2014). We continue to affirm the value of thoughtful, high-quality replication as a basic activity of a self-correcting and cumulative science, and as a vehicle for understanding the theoretical and methodological generality of effects.

Limitations of relying on individual study significance. The second standard's strict conventional criterion for significance, enforced study by study, has also led to a search for alternatives. The $p$ value is not a "truth statistic," even though the need for unambiguous standards of evidence has led to it being treated as one. A group of five true statements and one lie is more dishonest than a group of six true ones; but a group of five significant results and one nonsignificant is more to be expected than a group of six significant results, when sampling at $80 \%$ statistical power. Treating " $p<.05$ " as a gold standard of the truth makes two mistakes. 
First, it draws a rigid line across the spectrum of probability. This approach follows the deductive methods of hypothesis testing developed by Jerzy Neyman and Egon Pearson, rather than the inductive method that characterized Ronald Fisher's later writings (cf. Hurlburt \& Lombardi, 2009; Lehmann, 1993). However, Neyman-Pearson testing was developed with the aim of minimizing error over the long run, in a situation of quality control for instance. Fisher's aim of interpreting small numbers of individual results is more similar to the goal of research articles. In practice, most results in psychology have been reported using a hybrid of the two approaches that self-servingly maximizes positive interpretations: $p=.06$ is "marginally significant" and indicative of something Fisherian going on, while $p=.04$, just about as "marginal" in the other direction, is treated as solid proof. The alternative is to insist on the rigid criterion, but this leads to something worse: in a blinkered, absurd method of interpretation unworthy of thinking people, the $p=.07$ is taken as evidence for no actual effect and certainly something "different" from the $p=.04$. The critics of null-hypothesis significance testing are right in this: Something has to change about the way many people have used $p$ values.

Second, the "significant = true" fallacy puts too much faith in the significance of any one $p$ value as an indicator of underlying trends. Researchers underestimate the "dance of the pvalues," that is, the variation in the significance of tests of a true effect (Coulson, Healey, Fidler \& Cumming, 2010). A true effect, tested with good statistical power in proportion to its size, can yield a mixture of significance tests: many solidly significant, at $p<.01$; some in the marginal zone, from $p=.02$ to $p=.09$; and some non-significant with $p>=.10$, but still supporting the direction of the effect (Hung, O'Neill, Bauer, \& Kohne, 1997; Stanley \& 
Spence, 2014). If multiple experiments are required de facto to establish an effect, but only significant results are worthy of being reported, the unwillingness of reality to yield a significant result each time leads inevitably to unreported studies.

This "file drawer" of selectively unpublished, non-significant studies is one of the most vexing issues in research today. Some reasons to relegate a study to the drawer are legitimate: a well-identified error, or a manipulation that has not affected a measure of the independent variable. But to exclude data only because they do not support the narrative, with no independent reason, looks bad to laypeople and scientists alike. It is explicitly forbidden in the 6th edition of the Publication Manual of the American Psychological Association (2010); “Mention all relevant results, including those that run counter to expectations [...] Do not hide uncomfortable results by omission." (p. 32) The usual rationalizations of "streamlined" writing have a hollow echo to them. Journals don't have the page space to report dead ends? Not true in the era of online supplementary material. Readers would be bored or confused? Good writing can make an interesting story out of struggle; and in any case, readers of academic journals are supposed to be intellectuals, capable of handling a complex narrative.

The jury is still out on the exact impact of this practice on the truth value of the literature. Arguments have been made that even under publication bias, well-established directional results, if not exact effect sizes, are likely to hold (Fabrigar \& Wegener, in press; Murayama, Pekrun, \& Fiedler, 2013). But the doubt already cast on our results is considerable. Holding back on unfavorable evidence quite simply looks bad in the eyes of other scientists. 
Although it is unworkable to audit the file drawer literally, some patterns of results have the look of selective publication about them. Methods to interpret these patterns, such as the p-curve (Simonsohn, Nelson \& Simmons, 2014), are still under development and critique (Bishop \& Thompson, 2015; Ulrich \& Miller, 2015). It is not entirely clear how to use them to interpret the small numbers of focal results included in a typical single article. Showing faith in scientists' desire to tell a true story and not just a pretty one, a positive approach would offer encouragement to include reports of individual studies that fall short of significance but support an overall narrative, and even some mention of the reasons why "failed" studies are thought to have failed.

Limitations of focus on threats to positive results' validity. Our field has excelled at calling into question authors' interpretation of positive results, by bringing up potential confounds and alternative explanations. But ability to interpret negative results has been less well rewarded. For example, the typical published psychology study has had a low experimental power to detect any but a very large effect (Bakker, van Dijk \& Wicherts, 2012); published manipulations often lack check variables, pre-testing or calibration to the population sampled; low reliability of instruments is not an absolute barrier to publication; and finally, the extent to which effects appear across sets of stimuli is also left unclear from studies that rely on single examples (Judd, Westfall, \& Kenny, 2012). So, in the worst case, a negative result is hard to interpret, because we don't know whether to blame type II error, a manipulation that failed to take or to generalize, low reliability, or more interestingly, the absence or trivial size of the effect in the population. 
All these omissions are understandable in a field that has been focused on rewarding positive results. Why slow down your research with large samples, pre-testing, and so on? The obvious reason is to reduce the occurrence of false results, both positive and negative. But as I have argued, a way of doing research has taken hold that takes selective reporting of positive results for granted. Some even believe that finding significant results with unreliable methods means that the results are extra-strong and trustworthy, given the blurry microscope they are glimpsed through. But the concern is that the blurry microscope also runs the risk of letting us see results that aren't actually there, in the manner of a Rorschach test.

\section{New standards?}

The past few years in psychology have seen the crisis of confidence in the $p$-value reach a tipping point. Realizing that the $p$-value is not a truth-value, we need a clear criteria to replace it in judging the experimental evidence for a proposition. A number of journal editors have proposed to reduce or eliminate our reliance on $p$-values in favor of another kind of inferential statistics: confidence intervals, Bayesian, or none at all. These realizations have also increased the call for new criteria based on statistical power or sample size. However, in my view, the alternatives do not fully take into account the state of development of the theory and methods of social psychology.

Bayesian prior probabilities. As loannidis (2005) has argued, to turn the p-value into a truth value we need to also know the probability that the null hypothesis, as opposed to an alternative hypothesis, is true in the population. This is because, following Bayesian 
statistical logic, the p-value only tells us about the unlikeliness of the observed result if the null hypothesis holds in reality. An interactive visualization of these arguments and formulae can be found online (Schönbrodt, 2014; http://shinyapps.org/apps/PPV). Playing with various values, it becomes clear that the prior probability that a hypothesis is true outstrips statistical power in determining the truth-value of a given $p$-value.

For example, let's assume that our hypothesis is only directional (the experimental group has a higher score than the control group in the population) and that it has a $50 \%$ "coin flip" chance of being true. At $80 \%$ power, the chance that a positive result at alpha $=.05$ is true (positive predictive value, or PPV) is $94 \%$. At $35 \%$ power, we have less confidence in the truth of that result; the PPV is now $87.5 \%$, but this still means that the great majority of reported results are true. It is only when we turn to testing hypotheses with a very low likelihood of being true that we can agree with loannidis' titular claim that "most [positive] published research findings are false." With a hypothesis that is only $5 \%$ likely to be true, even $80 \%$ power yields a PPV just below $50 \%$, and $99 \%$ power barely gets us to a PPV of $51 \%$. Clearly, to be sure of the truth value of our positive findings, power alone will not save us. We have to see how likely they were in the first place. This in turn involves their proposed effect size; for example, the prediction that a tiny manipulation will lead to at least a tiny directional change is categorically more plausible than the prediction that the same manipulation will shift the outcome variable by one or more standard deviations. And of course, estimating effect size a priori is necessary to obtain power calculations in the first place. 
But the need to consider prior probabilities seems profoundly unfair, especially to a discipline that has grown up around the question of social prejudice. Surely every hypothesis deserves a fair trial on the basis of the evidence, regardless of what you might think beforehand? And yet, not all prejudices are irrational (Crandall \& Eshlemann, 2003). Our everyday decision-making, too, takes prior knowledge into account all the time. The real problem in accepting any kind of Bayesian criterion is our inability to quantify and agree upon the likely size of a novel experimental effect a priori. The vast majority of theories in social psychology continue generate only directional hypotheses, as Meehl (1967) argued. Without knowing on theoretical grounds the size of an entirely novel effect, we cannot calculate the chance of a Type II error, or properly specify the likelihood of any given observed result. Therefore, Bayesian and power calculations are most solid when following directly on already-obtained effects, such as when replicating other labs' experiments or confirming exploratory research. At other times, they have to proceed on the basis of a "typical" effect size for social psychology, or - in the case of Bayesian analyses - draw on non-informative or weakly informative priors (Gelman, 2009).

Effect size expectations. An often-cited effect size benchmark for social psychology is the conventionally small-to-medium mean effect size of $r=.21$ found by a meta-analysis of meta-analyses in the social psychology literature (Richard, Bond \& Stokes-Zoota, 2003). But in these meta-analyses we can see great variety in the questions asked, some irrefutable ("impressions are based on individuals' special characteristics", $r=.69$ ) and others debunking popularly held myths ("subliminal advertising increases sales", $r=.00$ ). Few of these meta-analysis topics correspond to the vital questions increasingly asked by 
experimental social psychologists over the past twenty years about the influence of mindsets, implicit processes, schemas, emotions, and goals. These topics, in fact, are often studied in idiosyncratic ways that don't lend themselves readily to meta-analysis.

Also importantly, the same question can be studied by many methods, and it is little appreciated that method can contribute greatly to observed effect sizes. For example, Abelson (1985) found that individual differences between batters contributed less than $1 \%$ of variance to the success of a single at-bat in Major League Baseball; but when studied as a more reasonable "repeated measures" design across multiple games and seasons, person factors assume their intuitively enormous role and explain nearly $49 \%$ of year-on-year variance in batting averages (Giner-Sorolla, unpublished, 2015). Richard et al. (2003) likewise found evidence that method and context factors contributed to variance in effect sizes, beyond the variance expected from mere resampling. More mature research topics paradoxically showed weaker overall effects, as experiments increasingly sought boundary conditions that would negate or reverse the finding. Should the purpose of research be to establish a method that maximizes basic effect sizes, or to find moderators and confounds that will tend to reduce them overall (and, as interaction effects, will have their own hardto-predict effect sizes?) This open question muddies the interpretation of existing effect sizes.

Effect sizes in basic research have a more fundamental problem. They are almost always based either on abstract measures (e.g., self-report scales, relative reaction times), or on concrete measures embedded in an abstract context, such as money earned in an economic 
game. Thus, effect sizes and related measures such as confidence intervals are hard to interpret. When comparing two conditions' responses on a seven-point mood scale, what does it mean to have a standardized effect size lower bound of .20, as opposed to .30 ? The fact that the larger lower bound is more strongly different from zero can also be conveyed by reporting the exact $p$-value. But comparisons to meaningful non-zero values are unlikely. The abstract nature of effect sizes in basic research makes it hard to use them in calculating cost-benefit tradeoffs, for example, or in relation to clinical criteria.

To be sure, effect size statistics are useful, and should be reported alongside significance. This makes the job of meta-analysis easier, and allows comparisons between multiple effects if they are methodologically similar. Effect sizes also are more meaningful when we measure concrete units in an ecologically valid setting, such as school test scores or clinically relevant levels of stress-related hormones. But these are not sufficient arguments to abandon $\mathrm{p}$-values in favor of confidence intervals or Bayesian statistics. Considering social psychology's directional hypotheses, and our often basic and abstract measures, a precisely reported and intelligently interpreted p-value can summarize directional evidence, balancing the magnitude, variability, and sample size of an effect.

\section{Explaining our new guidelines}

With this background in place, let me now annotate the new set of editorial policies and guidelines that can be found online at http://www.journals.elsevier.com/journal-ofexperimental-social-psychology/news/jesp-editorial-guidelines/, replacing the guidelines from 2014. 
1. All manuscripts must include a report of the following items:

- Explain how sample size was determined, including whether and how looking at the results influenced the collection of additional data. Reporting statistical power (together with the basis for expected effect size) is welcome, but not required.

- If participants were excluded from the study, report how many, how they were distributed across conditions, and the reasons for exclusion.

- Disclose the existence of all variables and conditions that were part of the study. These can be summarized, or put in a footnote or supplementary material in the case of large numbers of variables, but there should be enough information to judge whether they are potentially theoretically relevant or not.

- Affirm the above two disclosures positively (that is, with a statement such as "We report all measures, manipulations, and exclusions in these studies.")

- Report procedures in sufficient detail to allow close replication by an independent lab. This may include citations of method details found in other published, peerreviewed (or open access) sources. Materials are not required to be provided verbatim, but should be summarized with examples. To stay within the word limit of a report, necessary details can be included in Supplementary Materials.

These are the same requirements as before, with one change: papers will now have to include a positive affirmation that they have followed the previous requirements. This fits the goal to reduce the selective analysis of results and increase acceptance of a realistic 
picture of evidence. Authors should also note that the requirement to justify sample size does not rule out using fully reported, statistically corrected methods of sequential sampling (Lakens, 2014). This appears to be a promising way to establish a first estimate of an initially unknown effect size, avoiding much of the uncertainty around a priori power analysis.

2. All manuscripts should report complete statistics relevant to the analyses at hand, using supplementary materials if needed:

- cell means, SD and $n$ for experimental designs

- correlations between variables for multivariate designs including regression and repeated-measures

- inferential statistics with exact $p$-values and effect sizes regardless of significance level.

- If figures use error bars, these should be explained in a caption (e.g., standard error, 95\% confidence interval, etc.)

- If meeting any of these requirements proves impractical, the authors should explain why.

These requirements are expanded from the previous requirement to report effect size, because basic descriptive statistics are often critical to evaluating results (for example, examining multicollinearity in multivariate analyses, or floor or ceiling effects in variables). The importance of exact $p$-value has already been explained, while error bars are often misunderstood or underreported (Belia, Fidler, Williams \& Cumming, 2005). Often excluded from manuscripts in the interest of brevity, basic descriptive statistics can be 
included in Supplemental Materials, and will also be useful going forward in informing meta-analyses and other curations of the empirical literature.

3. FlashReports will now follow similar standards of evidence and potential topics as the other forms of articles, but will still be distinguished by a brief 2500 word limit and streamlined review process. The short format encourages research reports with background ideas and methods that do not require a great deal of explanation, but it does not mean that papers should be "short" on theoretical relevance or amount of evidence. Method and analysis details not crucial to the narrative of the paper should still be reported in Supplementary Materials.

4. Soliciting reviews of revised manuscripts is now the exception, not the rule. Associate Editors will typically come to a decision on a revised manuscript based on its capacity to address the reviewers' comments, unless expert opinions on technical matters are required. We expect this will make the review process faster and more efficient.

As explained, both these policies are part of the move to make the review process faster without compromising on quality.

5. Our standards for articles rest on a tripod of strong theory, strong methods, and strong statistical evidence for the points being made. Deficiencies in one of these areas can, to some extent, be compensated by strengths in the other two. 
Strong theories and strong methods - in terms of statistical power, consistency between studies, and clear distinction between exploratory and confirmatory tests - can improve the plausibility of reported results. A theory that convincingly explains unexpected findings, in fact, actually increases their subjective truth value; findings that are published merely because they are surprising, because they start from a low prior probability, are intrinsically less convincing. It is understood that a good paper for JESP will provide evidence not only that something happens, but also make at least a first attempt at explaining why. Strong theoretical relevance -- for example, a series of studies systematically pitting one theory's explanation of an effect against another -- is a highly desirable trait as well. If competing theories are well characterized and implemented, aggregate results need not be as strongly positive as when an author makes a one-sided theoretical argument.

The following guidelines (a-d) further explain how qualities of reported results can lead to a conclusion of strong statistical evidence.

a. In particular, our view of strong statistical evidence departs from the previous unwritten standard of "multiple studies, each with a significant effect." Instead, strong statistical evidence for a central positive claim now rests more upon showing a plausible pattern of results, usually from multiple comparable studies, whose total joint probability under the null would be about $p=.01$ or less (e.g., using Fisher's method to aggregate $p$ values from results in the same direction, or meta-analysis otherwise). However, we emphasize that this view is not a strict criterion. It does not prohibit publication of less strong results if theory and methods are both strong, nor 
is it a guarantee of publication if the article has other limitations. For example, although we continue to discourage submission of single-study articles because they often do not provide enough development and evidence for an idea, we are willing to consider them if based on good theory and exceptionally strong methods (possibly including: high power to detect a small-to-medium effect size, pre-registered methods and analyses, unusual or highly representative samples).

b. Within an article, individual studies with good theoretical and methodological connections to the others need not be statistically significant if they contribute to a strong overall pattern, a precise estimate, and/or a complete and open reporting of a program of research. Article-wise meta-analytic estimates are encouraged to aggregate the findings of multiple studies.

Following from the discussion of the nature and likely distribution of $p$-values, these items describe a new way to think about significance that roughly matches the previous implicit standard. For example, using Fisher's method, two independent results in the same direction at $p=.04$ have a joint value about $p=.011$. However, aggregate results that fall short of this standard can still be considered if they are theoretically wellgrounded and arise from strong and systematic methodology. I encourage authors to take a cumulative approach to summarizing their central findings across studies while also addressing their consistency, rather than feeling the need to only report results that look significant. 
c. Bayesian analyses with well-justified and well-explained priors can be used to assess evidence for reasonable null and alternative hypotheses.

Interesting answers to many questions can come as non-effects (such as "no gender difference in this experimental effect" or "no effect of subliminal advertising on consumer behavior") but the technique of arguing such a case statistically is not always known. Confidence in methodology that could have detected any reasonable effect "out there" should be high. Also, the question of which range of effect sizes should be treated as null needs to be considered as well. I believe that with these prior conditions, Bayesian analyses offer one way to balance evidence for a null and alternative hypothesis against each other, although the alternative hypothesis also needs to be specified with a reasonable range; overly strong alternative effect sizes tend to bias conclusions in favor of the null.

d. A pattern of multiple central results that are all below, but close to, $p=.05$, although they might have arisen by chance, also can be said to show low statistical robustness. Ideally, confidence in such a set of results can be bolstered by pre-registered studies or other methodological assurances against selective reporting.

Again, this is not a strict requirement but one of many considerations. I do realize that at a certain level of selective reporting, even an aggregate estimate becomes unreliable. While it has not been worked out yet in all its details, the p-curve and similar methodologies offer a simple insight: given the usual variability of $p$-values in a true effect, it is unlikely that a series of independent tests specified a priori would yield 
results all slightly under $p=.05$. Such results may be given greater scrutiny in terms of methodology and reporting. But rather than apply a hard-and-fast forensic rule, or require a lab-wise disclosure statement that would run into many problems defining the scope of a research line, I choose to start by encouraging authors to report consistent analyses and methods that support a larger picture, even if some individual results are not strictly significant. To this end, I also want to encourage authors to submit research articles based in part or fully on pre-registered studies; and to submit methods articles that develop, test, validate or criticize techniques for making decisions about the plausibility and robustness of a set of reported findings.

e. As before, we welcome rigorously conducted replication articles that meet the criteria described in Brandt, IJzerman, et al. (2014).

This guideline has not changed. If I could offer one further piece of advice for replication attempts, it is to consider carefully the pretesting, calibration, and validation of stimuli and contexts for the new sample, rather than just assuming that a duplication of the original procedure will stand as valid (cf. Fabrigar \& Wegener, in press). This is consistent with the reasons why original research should also pay more attention to calibration and validation.

6. Authors are encouraged to present exploratory work openly. It is deceptive to present hypotheses as perfectly precognitive when they aren't (i.e., avoid HARKing; Kerr, 1998). It is OK for authors to admit they entertained multiple hypotheses, based on 
multiple theoretical perspectives, without coming down on the side of any one of them or even to admit that they came down on the side of the wrong one! Put another way: an initial study, with an uncertain or novel prediction, should be treated as exploratory regardless of what story the authors choose to tell. Confidence in the results depends on sound methods, sample size, and consistent replication. Honest distinctions between exploratory and confirmatory work can be reported concisely without turning it into a long-winded "intellectual odyssey."

Research with strong and well-tested methods, like high-powered research, gives more credible positive findings. It also allows for evaluation of "failed" studies in a principled way, rather than assuming that their methods were faulty merely because they failed to support the preferred hypothesis (LeBel \& Peters, 2011). To this end, I encourage brief reporting (perhaps in footnotes or supplementary material) of interpretable failures or minor procedural tests along the way. Also, pre-registration of hypotheses and analyses offers one way to distinguish confirmatory from exploratory work. I urge those interested to watch for the upcoming special issue of JESP on Confirmatory Research for more examples and commentary on this practice.

7. Although mediation analyses are used in many, if not most, recent JESP articles, we urge greater caution in using and interpreting this technique (cf. Fiedler, Schott \& Meiser, 2011; Kline, 2015; Spencer, Zanna, \& Fong, 2005). As before, we see little value in mediation models in which the mediator is conceptually very similar to either the predictor or outcome. Additionally, good mediation models should have a 
methodological basis for the causal assumptions in each step; for example, when the predictor is a manipulation, the mediator a self-reported mental state, and the outcome a subsequent decision or observed behavior. Designs that do not meet these assumptions can still give valuable information about potential processes through correlation, partial correlation, and regression, but should not use causal language and should interpret indirect paths with caution. We reiterate that mediation is not the only way to address issues of process in an experimental design.

I have withdrawn the previous policy's suggestion, which was to test mediation models without clear causal direction by "reversing" the mediator and outcome to see which model seems stronger. Since then, it has been persuasively argued that this technique is influenced by artefacts such as measure reliability and does not necessarily indicate true underlying patterns of data (Lemmer \& Gollwitzer, 2016; Thoemmes, 2015). Many authors apparently think that mediation is a necessary requirement to be published. We want to dispel this misconception, and encourage alternate ways to show the role of third variables in experimental effects. A significant indirect path, where all three variables are measured at the same time and have no clear causal logic to them, can merely tell us that the "mediator" is not irrelevant to the "predictor," at the same time that it is not entirely redundant with the "predictor" in its relationship to the "outcome."

8. Arbitrary use of covariates can be used to engineer significant results. Therefore, covariates need to be justified as a response to a clear threat to validity. Reporting the uncovaried analysis can help clear this up (e.g., in a Footnote). 
9. Interaction effects on their own are not sufficient; they must be decomposed with simple effects analysis (Aiken \& West, 1991) or other means. At the same time, the direction and significance of simple effects are influenced both by interactions and main effects; therefore, it is not always necessary to "validate" an interaction by showing that both simple effects are significant (Petty, Fabrigar, Wegener, \& Priester, 1996).

These two points are unchanged from the 2014 guidelines.

What do the new standards look like in action? Perhaps one paper that I handled in my last year as Associate Editor can stand as a good example. Subscribers to the journal will be able to find Bostyn and Roets (in press), available in an online preprint version prior to the release of the March 2016 issue. In its final form, this article presents three studies, with somewhat different individual results, that give a strong and theoretically well-supported "big picture" of an asymmetry in the action-omission effect for positive and negative moral judgments. Pre-registered internal replication, aggregate meta-analysis across studies, and a Bayesian analysis using well-reasoned priors to support negative results in a particular condition, all form part of the new look of evidence. Going forward, I hope to see other manuscripts that take this approach to multi-study research.

In conclusion, I am sure that this Editorial will not be the last word during my term at JESP. I have learned so much from my own experience and the arguments of others already, and am prepared to learn more over the next three years. As always, before more change happens, I will endeavor to make sure it is understood and approved by the whole editorial team; and when it happens, I will be sure to let you know, online or in print. 
(c) 2016. This manuscript version is made available under the CC-BY-NC-ND 4.0 license http://creativecommons.org/licenses/by-nc-nd/4.0/

Giner-Sorolla JESP Editorial 24

Roger Giner-Sorolla

January, 2016 
References

Abelson, R. P. (1985). A variance explanation paradox: When a little is a lot. Psychological Bulletin, 97(1), 129-133.

American Psychological Association (2010). Publication manual of the American Psychological Association (6th ed.) Washington, DC: American Psychological Association.

Bakker, M., Dijk, A. van, \& Wicherts, J. M. (2012). The rules of the game called psychological science. Perspectives on Psychological Science, 7(6), 543-554.

Belia, S., Fidler, F., Williams, J., \& Cumming, G. (2005). Researchers misunderstand confidence intervals and standard error bars. Psychological Methods, 10(4), 389-396.

Bishop, D. V., \& Thompson, P. A. (2015). Problems in using p-curve analysis and text-mining to detect rate of p-hacking (No. e1956). PeerJ PrePrints. Retrieved from https://peerj.com/preprints/1266

Bostyn, D. H., \& Roets, A. (2016). The morality of action: The asymmetry between judgments of praise and blame in the action-omission effect. Journal of Experimental Social Psychology, 63, 19-25. Retrieved from http://www.sciencedirect.com/science/article/pii/S0022103115300172

Brandt, M. J., IJzerman, H., Dijksterhuis, A., Farach, F. J., Geller, J., Giner-Sorolla, R., ... Van't Veer, A. (2014). The replication recipe: What makes for a convincing replication? Journal of Experimental Social Psychology, 50, 217-224.

Coulson, M., Healey, M., Fidler, F., \& Cumming, G. (2010). Confidence intervals permit, but do not guarantee, better inference than statistical significance testing. Quantitative Psychology and Measurement, 1, 26.

Crandall, C. S., \& Eshleman, A. (2003). A justification-suppression model of the expression and experience of prejudice. Psychological Bulletin, 129(3), 414-446.

Fabrigar, L. R., \& Wegener, D. T. (in press). Conceptualizing and evaluating the replication of research results. Journal of Experimental Social Psychology.

Gabriel, K. R. (1978). A simple method of multiple comparisons of means. Journal of the American Statistical Association, 73(364), 724-729.

Gelman, A. (2009). Bayes, Jeffreys, prior distributions and the philosophy of statistics. Statistical Science, 24(2), 176-178. 
Giner-Sorolla, R. (2015). Effect size depends on methods. Unpublished manuscript, University of Kent. Retrieved from https://osf.io/w4enh/.

Hewstone, M., \& Stroebe, W. (2008). Moving at snail's pace: Some observations on the publication process in social and personality psychology. SPSP Dialogue, 23(1), 17-24.

Hung, H. M. J., O'Neill, R. T., Bauer, P., \& Kohne, K. (1997). The behavior of the $p$-value when the alternative hypothesis is true. Biometrics, 53(1), 11-22.

Hurlbert, S. H., \& Lombardi, C. M. (2009). Final collapse of the Neyman-Pearson decision theoretic framework and rise of the neo-Fisherian. Annales Zoologici Fennici, 46, 311-349.

loannidis, J. P. A. (2005). Why most published research findings are false. PLoS Medicine, 2(8), e124.

Judd, C. M., Westfall, J., \& Kenny, D. A. (2012). Treating stimuli as a random factor in social psychology: a new and comprehensive solution to a pervasive but largely ignored problem. Journal of Personality and Social Psychology, 103(1), 54-69.

Lakens, D. (2014). Performing high-powered studies efficiently with sequential analyses. European Journal of Social Psychology, 44(7), 701-710.

Ledgerwood, A., \& Sherman, J. W. (2012). Short, sweet, and problematic? The rise of the short report in psychological science. Perspectives on Psychological Science, 7(1), 60-66.

Lehmann, E. L. (1993). The Fisher, Neyman-Pearson theories of testing hypotheses: One theory or two? Journal of the American Statistical Association, 88(424), 1242-1249.

Lemmer, G. \& Gollwitzer, M. (2016). The "true" indirect effect won't (always) stand up: When and why reverse mediation testing fails. Unpublished manuscript, Philipps-University Marburg.

Meehl, P. E. (1967). Theory-testing in psychology and physics: A methodological paradox. Philosophy of Science, 34, 103-115.

Murayama, K., Pekrun, R., \& Fiedler, K. (2013). Research practices that can prevent an inflation of false-positive rates. Personality and Social Psychology Review, 18(2), 107-118.

Richard, F. D., Bond Jr, C. F., \& Stokes-Zoota, J. J. (2003). One hundred years of social psychology quantitatively described. Review of General Psychology, 7(4), 331-363.

Schönbrodt, F. (2014). When does a significant p-value indicate a true effect? Web page, retrieved from http://shinyapps.org/apps/PPV. 
Simonsohn, U., Nelson, L. D., \& Simmons, J. P. (2014). P-curve: A key to the file-drawer. Journal of Experimental Psychology: General, 143(2), 534-547.

Spellman, B. A. (2015). A short (personal) future history of Revolution 2.0. Perspectives on Psychological Science, 10(6), 886-899.

Stanley, D. J., \& Spence, J. R. (2014). Expectations for replications: Are yours realistic? Perspectives on Psychological Science, 9(3), 305-318.

Thoemmes, F. (2015). Reversing arrows in mediation models does not distinguish plausible models. Basic and Applied Social Psychology, 37(4), 226-234.

Ulrich, R., \& Miller, J. (2015). p-hacking by post hoc selection with multiple opportunities: Detectability by skewness test?: Comment on Simonsohn, Nelson, and Simmons (2014). Journal of Experimental Psychology: General, 144(6), 1137-1145. 